\title{
Bei Trigeminusneuralgie wirksamer als Placebo
}

\section{Hier steht eine Anzeige.}

Fragestellung: Ist BIIB074 ein Nav1.7-selektiver Natriumkanalblocker bei Patienten mit Trigeminusneuralgie wirksam?

Hintergrund: Die Trigeminusneuralgie ist eine typische Alterserkrankung. Therapiestandards sind Carbamazepin und Oxcarbazepin, die als spannungsabhängige Natriumkanalblocker wirken. Die Wirksamkeit der beiden Substanzen ist unbestritten, wobei bei älteren Menschen die Nebenwirkungen eine besondere Rolle spielen. Daher besteht die dringende Notwendigkeit neue Substanzen zur Behandlung der Trigeminusneuralgie zu entwickeln, die weniger Nebenwirkungen als Carbamazepin und Oxcarbazepin haben. Nav1.7 ist ein Natriumkanal, der auf trigeminalen Neuronen exprimiert wird. Menschen, die eine reduzierte Kanalfunktion aufweisen, sind weniger empfindlich gegenüber experimentellen Schmerzen. Ziel der vorliegenden Studie war es, die neue Substanz bei Patienten mit Trigeminusneuralgie zu untersuchen.

Patienten und Methodik: Die doppelblinde multizentrische placebokontrollierte, randomisierte Phase-IIa-Studie wurde in 25 Schmerzzentren in Europa und Südafrika durchgeführt. Es wurden Patienten mit Trigeminusneuralgie im Alter zwischen 18 und 80 Jahren eingeschlossen, die zunächst alle für 21 Tage dreimal täglich $150 \mathrm{mg}$ BIIB074 erhielten. Patienten die auf die Therapie ansprachen, wurden dann für die nächsten 28 Tage in einem doppelblinden Design mit BIIB074 weiterbehandelt oder sie erhielten Placebo. Der primäre Endpunkt der Studie war die Zahl der Patienten, die in der doppelblinden Behandlungsphase als Therapieversager eingeschätzt wurden. Daneben wurde auch die Sicherheit der Substanz beurteilt.

Ergebnisse: Insgesamt wurden 67 Patienten in dem offenen Teil der Studie eingeschlossen und 30 Patienten randomisiert. Während der doppelblinden Behandlungsphase waren fünf Patienten (33\%) in der Verumgruppe und neun (64\%) in der Placebogruppe Therapieversager. Der Unter-

Zakrzewska JM, Palmer J, Morisset $\mathrm{V}$ et al. Safety and efficacy of a Nav1.7 selective sodium channel blocker in patients with trigeminal neuralgia: a doubleblind, placebo-controlled, randomised withdrawal phase $2 a$ trial. Lancet Neurol 2017; 16: $291-300$ schied war statistisch nicht signifikant. BIIB074 wurde hervorragend vertragen und die Rate an Nebenwirkungen unterschied sich nicht von der von Placebo. Die häufigsten Nebenwirkungen waren Kopfschmerz gefolgt von Benommenheit.

Schlussfolgerungen: In einer kleinen placebokontrollierten Studie war der selektive Natriumkanalblocker BIIB074 wirksamer als Placebo.

\section{- Kommentar von Hans-Christoph Diener, Essen}

\section{Größere Studien sind gerechtfertigt}

Die vorliegende Studie hat Hinweise darauf gegeben, dass BIIB074 bei Patienten mit Trigeminusneuralgie wirksam sein könnte. Die Fallzahl war allerdings deutlich zu gering um einen statistischen Behandlungseffekt zu zeigen. Die Verträglichkeit der Substanz war sehr gut, sodass es gerechtfertigt ist, mit dieser Substanz größere Studien bei älteren Menschen mit Trigeminusneuralgie durchzuführen. Besonders geeignet sind diese Studien bei Patienten die Carbamazepin oder Oxcarbazepin wegen Nebenwirkungen nicht tolerieren. 